\title{
Melanotic neuroectodermal tumour of infancy: A case report and review of the aetiopathogenic hypotheses
}

\author{
Carlos Madrid ${ }^{1}$, Jacqueline Aziza ${ }^{3}$, Anasse Hlali ${ }^{4}$, Kahina Bouferrache ${ }^{2}$, Marcelo Abarca ${ }^{2}$ \\ ${ }^{1}$ Head Department of Oral Surgery, Oral Medicine and Dental Care, University of Lausanne School of Medicine, Switzerland \\ ${ }^{2}$ Consultant, Department of Oral Surgery, Oral Medicine and Dental Care, University of Lausanne School of Medicine, Swit- \\ zerland \\ ${ }^{3}$ Anatomopathologist, Department of Pathology, Purpan Hospital, University of Toulouse, France \\ ${ }^{4}$ Dental Surgeon, Division Oral Surgery and Medicine, Hôtel Dieu Hospital, Toulouse, France
}

Correspondence:

Department of Oral Surgery

Oral Medicine and Dental Care

University of Lausanne

44 Bugnon Street,

1011 Lausanne, Switzerland

carlos.madrid@hospvd.ch

Received: 07/09/2009

Accepted: $24 / 12 / 2009$

\author{
Madrid C, Aziza J, Hlali A, Bouferrache K, Abarca M. Melanotic neu- \\ roectodermal tumour of infancy: A case report and review of the aetio- \\ pathogenic hypotheses. Med Oral Patol Oral Cir Bucal. 2010 Sep 1;15 \\ (5):e739-42. \\ http://www.medicinaoral.com/medoralfree01/v15i5/medoralv15i5p739.pdf

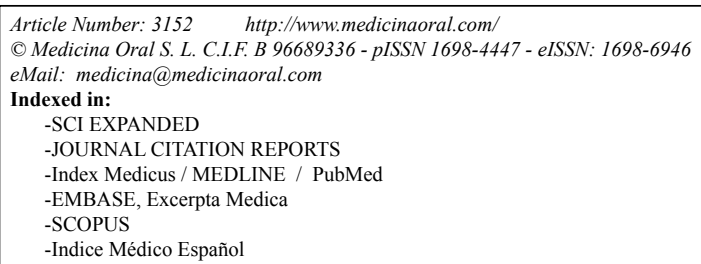

\begin{abstract}
The case of a 2-month-old healthy infant without relevant medical history. The patient was referred due to the aggravation of a swelling occupying the left half of the anterior maxilla. This lesion became visible approximately one month ago; it involved the buccal gingiva and alveolar bone, including the deciduous tooth germs 6.1 and 6.2. The swelling had dimensions of $20 \mathrm{~mm}$ x $20 \mathrm{~mm}$. The surgical excision was performed under general anesthesia. The tooth buds of 6.1 and 6.2 were closely related to the tumour and so were removed. The lesion was entirely enucleated. The pathology of the lesion confirmed a melanotic neuroectodermal tumour of infancy. The melanotic neuroectodermal tumour of infancy (MNTI) has been described as a rare benign pigmented painless swelling that usually occurs in the anterior region of the maxilla and in the incisor region. The histological examination showed small basophilic cells, many containing melanin pigmentation within the cytoplasm, with a second population of larger cubical cells with abundant cytoplasm, arranged in alveolar or adenoid clusters. According to Krompecher this tumour derives from epithelial nests evolved at the time of embryonic fusion of the facial processes. It has also been suggested that the tumour arises from the retinal anlage by a pinching-off process of neuroepithelium during the formation of embryonic eye. More recently, the presence of high levels of vanillylmandelic acid suggest a neural origin of the tumour.
\end{abstract}

Key words: Melanotic neuroectodermal tumour, melanine, maxilla. 


\section{Introduction}

The melanotic neuroectodermal tumor of infancy is a rare clinical entity, non ulcerative fast-growing tumefaction, located in the head and neck of children younger than 1 year. It's a pigmented tumor, although the pigmentation cannot always be observed through the covering tissues. The terminological variations undergone by this entity with 23 different denominations reported illustrates the uncertainties about its histogenesis. For some time, it was topographically related to the teeth in the vicinity of which it develops during the first year of life: it was then known under the term of melanotic adamantinoma.

The term of "retinal anlage tumour" was proposed by reference to the assumption of a haemartomatous tumour of retinal origin (1). In 1966, Borello and Gorlin reported a case characterised by a high urinary excretion of vanillylmandelic acid classically found in a phaeochromocytoma as well as in other neuroectoblastic tumours (retinoblastoma, ganglioneuroblastoma, neuroblastoma) suggesting a neuroectodermal origin for this lesion (2). Based on this, the proposed term of neuroectodermal melanotic tumour of infancy was currently retained by the WHO.

\section{Case Report}

The patient, a 2-month old infant, with no relevant medical history, was referred due to the aggravation of a swelling occupying the left half of the anterior maxilla. The parents complained about the increasing problems their child was having while eating. This lesion became visible approximately one month ago; it involved the buccal gingiva and alveolar bone, including the deciduous tooth germs 6.1 and 6.2. The swelling had dimensions of $20 \mathrm{~mm} \times 20 \mathrm{~mm}$. The intra-oral examination showed a round mass, of bluish colour, covered by the mucous membrane. The lesion was pedunculated (Fig. 1). The mass was resilient and painless. The CT Scan (Fig. 2) showed a cystic-shaped lacuna developed within the anterior maxilla, with well demarcated limits, expanding to the buccal cortex without perforation.

The contents of the lesion had the same density as the surrounding soft tissues. The lesion was pushing the sourrounding dental germs in a palatal direction, except for the tooth 6.1, which was included in the lacuna. No other maxillofacial localizations of the tumour were observed in this infant.

The surgical excision was performed under general anesthesia. The tooth buds of 6.1 and 6.2 were closely related to the tumour and so were removed. The lesion was entirely enucleated. A second curettage was performed to eliminate in particular a dark coloured area of bone around the tooth bud of 6.3. The patient's recovery was uneventful. There were no post-operative complications and no clinical recurrence (Fig. 3).

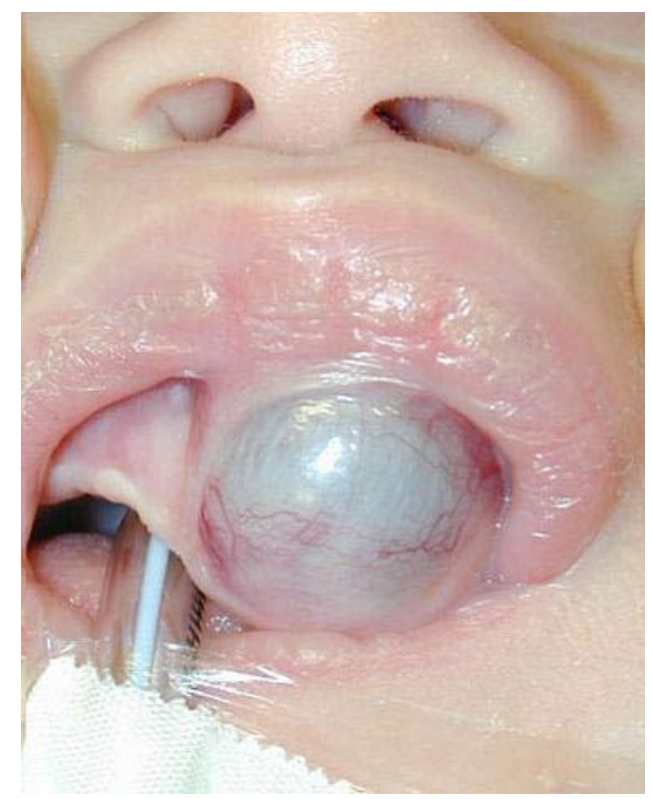

Fig. 1. Clinical view of the tumour.

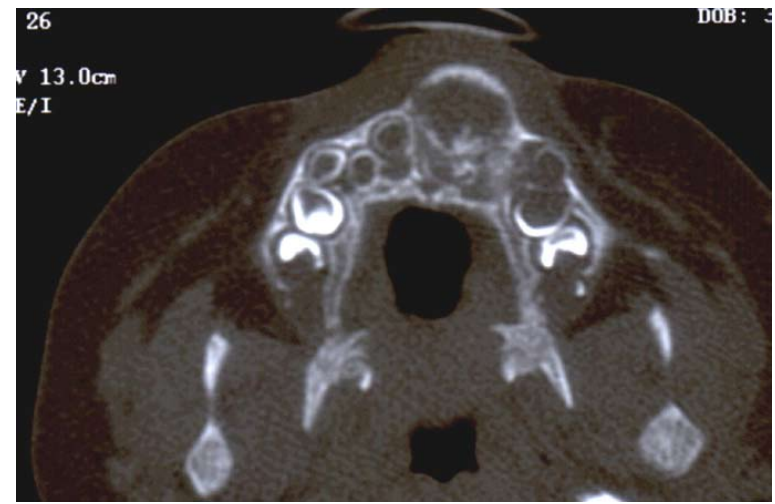

Fig. 2. CT Scan of the maxillary bone.

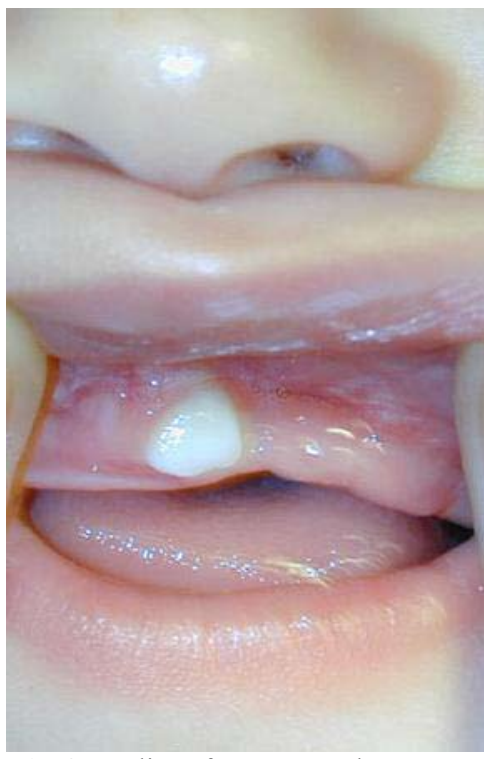

Fig. 3. Healing after one month. 
The pathology of the lesion confirmed a melanotic neuroectodermal tumour of infancy (MNTI). The tumour was formed by two cellular types arranged in a rich connective-fibrous stroma. In one respect, there were epithelial-like cells, arranged in small islands, outlining acinous or glandular structures. These large cells showed a finely rounded nucleolus core and had an abundant cytoplasm rich in melanotic pigments. In another respect, there were, less differentiated neuroblastlike cells, resembling those observed in neuroblastomas, with a round hyperchromatic core, and a poorly visible cytoplasm. This second cell population was generally grouped in independent, but not very cohesive, small islands or occasionally arranged in bunches, and surrounded by pigmented epithelioid cells. There were cellular crushing features as in neuroendocrine tumours. The cytonuclear anomalies were discrete and there were no mitosis. An immunohistochemical study was also conducted to specify the immunophenotypic profile. Because of the strong-pigmentation of the lesion, the alkaline phosphatase technique was used.

\section{Discussion}

This lesion is rare; no more than 365 cases were reported in the literature till October 2008 (3). The MNTI has no gender predilection and affects generally infants between 3 to 6 months: $43 \%$ of the cases of Cutler and co-workers, and $77 \%$ of the patiens were younger than 6 months in the serie of Kruse-Lösler et al. (4). Some cases have been reported in adults (5). Gender preference is still a matter of debate: the mayority of the publications report no difference, although Kruse-Lösler et al. reports an higer prevalence of the lesion in men (male/female: 1.48). In more than $90 \%$ of the cases the lesion is localized in the maxilla. Other common localizations are: the skull, the mandible and the brain, but cases in the epididymis, the mediastinum, and the foot have also been reported (4). This lesion is regarded as benign, although it can show locally aggressive behaviour, with gradual invasion of the surrounding bone and the sinuses. Recurrences have been described after surgical excision, and in exceptional cases, metastasis have been discovered (6). The rate of recurrence has been estimated at $15 \%$ and the rate of malignant transformation at $6.6 \%$ (4).

The recurrences reported could be the consequence of an incomplete remotion of the tumour (3). According the data of the literature, the rate of malignisation has been estimated to be arround 6.5\% (4). This rate is much higher than the observed for other melanotic tumours of the skin. However, the risk of malignant transformation might be exagerated in the literatura because malignant cases of MNTI are more suitable for publication than benign ones.
Etiopathogenic hypotheses:

According to Krompecher, this tumour derives from epithelial islands trapped during the embryonic fusion of the facial buds, but their aggressive behaviour urged him to propose the Latin term of "melanocarcinoma congenitum processus alveolaris". Halpert et al. Suggested later that the tumour arises from the retinal anlage by a process of pinching off of the neuroectoblast eye (1). This receives some support because the cytokeratines 7,8,18 and 19 are expressed by cultures of retinal pigmentary cells by the MNTI (7). The assumption of a pineal origin of the MNTI was advanced on the basis of histomorphological resemblance between this tumour and the tissue organization of the fetal pineal gland (8). Currently, the Borello et al. assumption prevails, based on the regular, although inconstant, significant rise in the rate of urinary excretion of vanillylmandelic acid (VMA) associated to MNTI (2).

VMA is a major urinary catabolite of catecholamines. Its free form found in great quantity is regarded as a marker of the neuroectodermal tumours. The disappearance of high rates of VMA after MNTI surgical removal confirms the neurocristopathic hypothesis (6). Nevertheless, a significant number of cases of MNTI associated with normal levels of VMA appear in the literature. However, a negative rate of VMA should not influence the diagnosis of MNTI, because not all the cells of the neural crest are involved in the metabolism of catecholamines.

The histochemical and ultra structural features of MNTI cells make them very similar to the neural crest cells (9). The large cells with melanin resemble a neuroepithelium, while the small non-pigmented cells resemble immature neuroblasts, or differentiating neuroblasts (10). All the cellular types clearly have ultra structural features specific to the neurogenic cells.

This accumulation of arguments supports the neural crest origin of the MNTI. The discovery of neuroectodermal cells in the areas where the tumour is more often encountered leads the proposition of its origin on the neural crest, the retinal anlage, the pineal anlage or the vomeronasal Jacobson's organ. Actually, taking into account the frequency of MNTI in the jawbones, the neural crest cells destined to the dental lamina should be considered in the first place. A differentiation pathway, under mesenchymal direction, could influence the tumour stroma morphogenesis: by a progressive transition from isolated fusiform cells within common fibroblasts to clusters of large pigmented cells (11).

Finally, MNTI seems to belong to the category of primitive neuroectodermal tumors of infancy which includes neuroblastoma, the peripheral neuroepithelioma [PNET], the malignant thoracopulmonar small round cell tumour, and some examples of Ewing's sarcoma. The relation with this group is confirmed by the isola- 
tion of a cellular line of PNET from a human primitive MNTI and grafted on mice.

Radiological aspects:

Conventional radiographs and CT images are useful (but not specific) for assessment of the lesion extension.

The usual image is a large cavity of osteolysis with well-delineated form without any specificity. The image could be less marked, from a simple thinning of the cortical to real bone destruction, with or without osteogenic reaction. The margins of the radiolucency displace the adjacent bone and tooth buds suggesting a tumour-like invagination inside the dental follicle (12). This image poorly delineated, with heterogeneous contents organised in wheel spokes, could lead to the diagnosis of an osteogenic sarcoma.

The MRI with gadolinium enhancement gives the clearest images, allowing the distinction of the tissue components of this tumour. The MRI reveals a hypodense mass, within a heterogeneous well-delineated lesion on T1- and T2-weighted images. The empty circulatory areas observed in hemangiomas are not present in the MNTI.

\section{Surgical management:}

Due to the accepted benign nature of this tumour, most authors agree on a conservative surgical treatment. In case of recurrence, extensive resection associated with reconstructive surgery is indicated. A radical, not too mutilating surgery in young patients is one of the most challenging parts of the treatment (13).

The clinical monitoring of the patients and radiological survey are essential. When a metastatic dissemination arises, the secondary lesions are only made up of few differentiated cells of the neuroblastoid line, which is regarded as the aggressive component of the tumour. Chemotherapy is not a usual treatment procedure, except for cases of confirmed metastatic diffusion. At the cellular level, the result of the chemotherapy shows an impressive reduction of the neuroblastoid population (14). The malignant change of these tumours remains unexplained. The potential of recurrence or metastatic dissemination cannot be inferred from the macro or microscopic features of the tumour.

\section{References}

1. Halpert B, Patzer R. Maxillary tumor of retinal anlage. Surgery. 1947;22:837-41.

2. Borello ED, Gorlin RJ. Melanotic neuroectodermal tumor of infancy--a neoplasm of neural crese origin. Report of a case associated with high urinary excretion of vanilmandelic acid. Cancer. 1966;19:196-206.

3. Neven J, Hulsbergen-van der Kaa C, Groot-Loonen J, de Wilde PC, Merkx MA. Recurrent melanotic neuroectodermal tumor of infancy: a proposal for treatment protocol with surgery and adjuvant chemotherapy. Oral Surg Oral Med Oral Pathol Oral Radiol Endod. 2008;106:493-6.

4. Kruse-Lösler B, Gaertner C, Bürger H, Seper L, Joos U, Kleinheinz J. Melanotic neuroectodermal tumor of infancy: systematic re- view of the literature and presentation of a case. Oral Surg Oral Med Oral Pathol Oral Radiol Endod. 2006;102:204-16.

5. Calabrese F, Danieli D, Valente M. Melanotic neuroectodermal tumor of the epididymis in infancy: case report and review of the literature. Urology. 1995;46:415-8.

6. Johnson RE, Scheithauer BW, Dahlin DC. Melanotic neuroectodermal tumor of infancy. A review of seven cases. Cancer. 1983;52:661-6.

7. Hunt RC, Davis AA. Altered expression of keratin and vimentin in human retinal pigment epithelial cells in vivo and in vitro. J Cell Physiol. 1990;145:187-99.

8. Dooling EC, Chi JG, Gilles FH. Melanotic neuroectodermal tumor of infancy: its histological similarities to fetal pineal gland. Cancer. 1977;39:1535-41.

9. Nikai H, Ijuhnin N, Yamasaki A, Niitani K, Imai K. Ultrastructural evidence for neural crest origin of the malanotic neuroectodermal tumor of infancy. J Oral Pathol. 1977;6:221-32.

10. Claman LJ, Stetson D, Steinberg B, Shuler CF. Ultrastructural characteristics of a cell line derived from a melanotic neuroectodermal tumor of infancy. J Oral Pathol Med. 1991;20:245-9.

11. Kaya S, Unal OF, Saraç S, Gedikoğlu G. Melanotic neuroectodermal tumor of infancy: report of two cases and review of literature. Int J Pediatr Otorhinolaryngol. 2000;52:169-72.

12. Hoshina Y, Hamamoto Y, Suzuki I, Nakajima T, Ida-Yonemochi $\mathrm{H}$, Saku T. Melanotic neuroectodermal tumor of infancy in the mandible: report of a case. Oral Surg Oral Med Oral Pathol Oral Radiol Endod. 2000;89:594-9.

13. Shaia WT, Dinardo LJ, Underhill TE, Cesca CE. Recurrent melanotic neuroectodermal tumor of infancy. Am J Otolaryngol. 2002;23:249-52.

14. Mello RJ, Vidal AK, Fittipaldi HM Jr, Montenegro LT, Calheiros LM, Rocha GI. Melanotic Neuroectodermal Tumor of Infancy: Clinicopathologic Study of a Case, with Emphasis on the Chemotherapeutic Effects. Int J Surg Pathol. 2000;8:247-251. 